\title{
Acoustic Emission Location Experiment Research on Stope Roof Breakage Boundaries Time-Varying Process
}

\author{
Liang Zhong-Yu ${ }^{*}{ }^{1,2}$, Chen Zhan-Qing ${ }^{1}$, Gao Feng ${ }^{1}$ and Zhou Yu-xin ${ }^{2}$ \\ ${ }^{1}$ State Key Laboratory For Geomechanics \& Deep Underground Engineering, CUMT, Xuzhou, Jiangsu, 221008, China \\ ${ }^{2}$ Department of Basic Courses, Military Traffic Institute, Tianjin,300161, China
}

\begin{abstract}
This paper utilizes self-designed time-varying boundary sound emission test system for rock stratum fracture to complete the sound emission orientation test upon the fracture of marble slab. A new method-image diagnosis and orientation method is proposed to locate fracture section. Sound emission signal Y Position and Events-X position distribution point diagram is utilized to determine the position of fracture surface; by analyzing the time-varying curve of Counts, Energy and Hits of acoustic emission signal in the same stage, the occurrence moment of rock stratum fracture section is confirmed and the development mechanism of fracture section is analyzed. Through study, it can be concluded that located fracture section position is close to actual fracture position, indicating that sound emission image diagnosis technique is characterized by high orientation precision, small error and good orientation effect; sound emission image diagnosis orientation method has very excellent maneuverability and practicability.
\end{abstract}

Keywords: Acoustic emission, positioning, roof fracture, rock damage, system with variable boundary.

\section{INTRODUCTION}

During the growth process of internal defects, solid materials release energy which transmits to the surroundings in the form of elastic waves [1,2]; growth point of internal defect is called as sound emission source. During the detection of sound emission, the method to integrate the parameter characteristics of sound emission signal detected by sensors and determine the position of sound emission source is called as source orientation [3, 4]. Source orientation is one of the important functions of sound emission technique and its common types are shown in Table 1.

Table 1. The source position classification.

\begin{tabular}{|c|c|c|c|}
\hline Source Type & \multicolumn{3}{|c|}{ Orientation Method } \\
\hline \multirow{5}{*}{ Burst type } & \multirow{3}{*}{$\begin{array}{l}\text { Time difference } \\
\text { orientation }\end{array}$} & 1D orientation & Plane orientation \\
\hline & & $2 \mathrm{D}$ orientation & Cylinder orientation \\
\hline & & 3D orientation & Sphere orientation \\
\hline & \multirow{2}{*}{$\begin{array}{l}\text { Regional } \\
\text { orientation }\end{array}$} & \multicolumn{2}{|c|}{ Independent channel monitoring } \\
\hline & & \multicolumn{2}{|c|}{ Signal arrival sequence } \\
\hline \multirow{4}{*}{$\begin{array}{l}\text { Successive } \\
\text { type }\end{array}$} & \multicolumn{3}{|c|}{ Amplitude measuring-type area orientation } \\
\hline & \multicolumn{3}{|c|}{ Attenuation measuring-type orientation } \\
\hline & \multicolumn{3}{|c|}{ Mutual correlation time-difference orientation } \\
\hline & \multicolumn{3}{|c|}{ Interference time-difference orientation } \\
\hline
\end{tabular}

*Address correspondence to this author at the State Key Laboratory For Geomechanics \& Deep Underground Engineering, CUMT, Xuzhou, Jiangsu, 221008, China; E-mail: liang_zhongyu@163.com
Continuous signal source orientation is mainly for the orientation of liquid or gas leakage source. Stope roof is mostly made of hard rock whose sound emission source comes out as a burst type. Therefore, this article concentrates on the orientation of burst-type signal source.

According to the self-developed stratum fracture timevarying boundary sound emission test system, this paper carried out the test and study on boundary time-varying roof fracture orientation with sound emission technique. Marble slab has been utilized to make the roof of stope. Multiple orientation techniques for sound emission orientation have been applied, such as time-difference method, threshold value method and maximum energy method [5]. It is the first time to introduce image diagnosis orientation method to determine the time and space position of $\mathrm{AE}$ (Acoustic Emission) source and extend AET orientation method.

\section{IMAGE DIAGNOSIS ORIENTATION TECHNIQUE}

\subsection{Proposing of Image Diagnosis Orientation Technique}

Computer is utilized for processing of image information, including noise elimination, enhance-ment, recovery, segmentation, characteristic extraction and so on, and then re-analyze the processed image information [6]. This analysis process is known as image diagnosis.

Development of image diagnosis technique is mainly affected by 3 factors: first, computer development; second, mathematical development; third, a wide range of application demands in husbandry, forestry, environment, military, industry and medicine.

Image diagnosis technique has been widely applied in many fields of national economy. Staff of agriculture and 
forestry department keeps track of the growth of plants, evaluates the production and monitors the plant diseases and insect pests through the analysis on remote sensing images. Department of water conservancy can get to know the change in water disaster and flood through the analysis on remote sensing images [7]. Meteorological department can improve the precision of weather forecast by meteorological map. Departments of national defense and mapping can obtain the information of territory, landform and facilities on the ground through aerial survey or satellite observation. Mechanical department utilizes image processing technique to automatically analyze and identify constitution diagram. Medical image department performs disease diagnosis through digital image information processing, for example, the ultrasonic inspection.

To expand the application and field of sound emission technology, and solve the problems of material injury or complicated structural injury, the attempt is made to apply image diagnosis technology to sound emission orientation test. Image diagnosis sound emission orientation technology is utilized to determine the fracture position and time of roof during time-varying boundary process [8]. Sound emission image diagnosis and orientation technology provide new ideas to the solution of other practical engineering problems.

\subsection{Application of Image Diagnosis Orientation Technology}

To finally determine the position of sound source, image diagnosis sound emission orientation technology needs to analyze multiple sound emission signal curves and integrate the characteristics of various sound emission signals. Detailed operation is as follows:

(1) Proper methods are utilized to correctly collect the sound emission parameter information of oriented objects.

(2) Digital image processing function of sound emission software is applied to process sound emission parameter information of oriented objects and obtain corresponding image information. For example, it can be applied to determine sound emission event point diagram of sound emission source position, sound emission wave hits of material injury characteristics, ringing counting and energy curve during characteristic sound emission test.

(3) By incorporating physical characterization significance of sound emission parameters, each sound emission image is analyzed, combining the analytical results of images, and finally obtaining a reasonable orientation conclusion.

\section{BOUNDARY TIME-VARYING SOUND EMISSION ORIENTATION TEST FOR STOPE ROOF FRACTURE WITH MARBLE SLAB SIMULATING STOPE ROOF}

\subsection{Orientation Test}

According to requirement of orientation test, $4150 \mathrm{KHz}$ resonance sensors are fixed to the predetermined position of marble slab with Vaseline as coupling agent and sellotape, as shown in Figs. (4-6). The gain of pre-amplifier is adjusted to $40 \mathrm{~dB}$ and 4 sensors are connected to the channel 1,2, 3 and 4 of appliance (See Fig. 1).

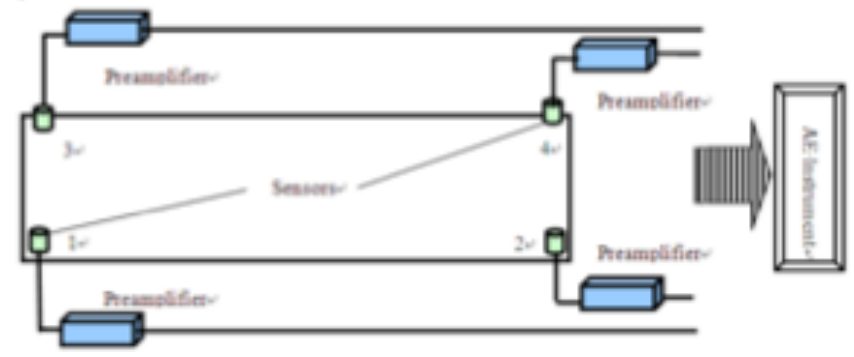

Fig. (1). Sensor layout.

Orientation test equipment works on self-developed stratum fracture boundary time-varying sound emission test system. Simulated stratum is a marble stratum sized $1300 \times 195 \times 20$. Orientation test adopts different unloading pressure maintaining schemes with 5 groups in all. The orientation test equipment collects and records the Time, Amplitude, Events, Energy, Hits, Counts, Duration, ASL and Risetime of sound emission.

As each group of simulated roof boundary time-varying orientation test is characterized by long time history, large volume of sound emission signal and complicated operation procedure, test process and data collection are conducted /in steps.

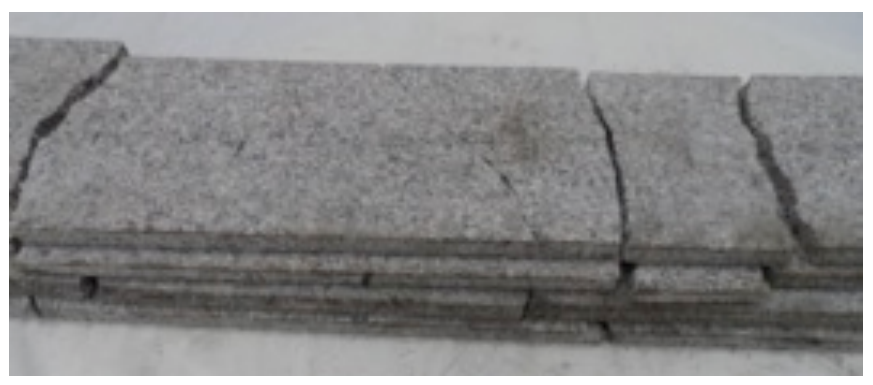

Fig (2). Rock fracture images.

The image of fractured marble stratum in orientation test is shown in Fig. (2). As the shrinking of supporting cylinder and data collection is conducted at one stage, data orientation analysis and treatment should be conducted at a stage too. The orientation result of fracture section is compared with actual position of fracture section to obtain corresponding conclusion and rule.

\subsection{Image Diagnosis Analysis}

Next, image diagnosis analysis is conducted on marble stratum of one simulated stratum and the image information of one stage. The analysis of other stratums and stages then works similarly.

\subsubsection{Amplitude-time curve}

Fig. (3) shows the time-varying curve of AE signal amplitude for each channel. This curve shows the intensity of each channel signal in different times and one can observe if there is single signal during the test. It can be seen from Fig. (3) that channel 1 has abundant signals, but with lower amplitude; channel 2 has generally lower AE signal 


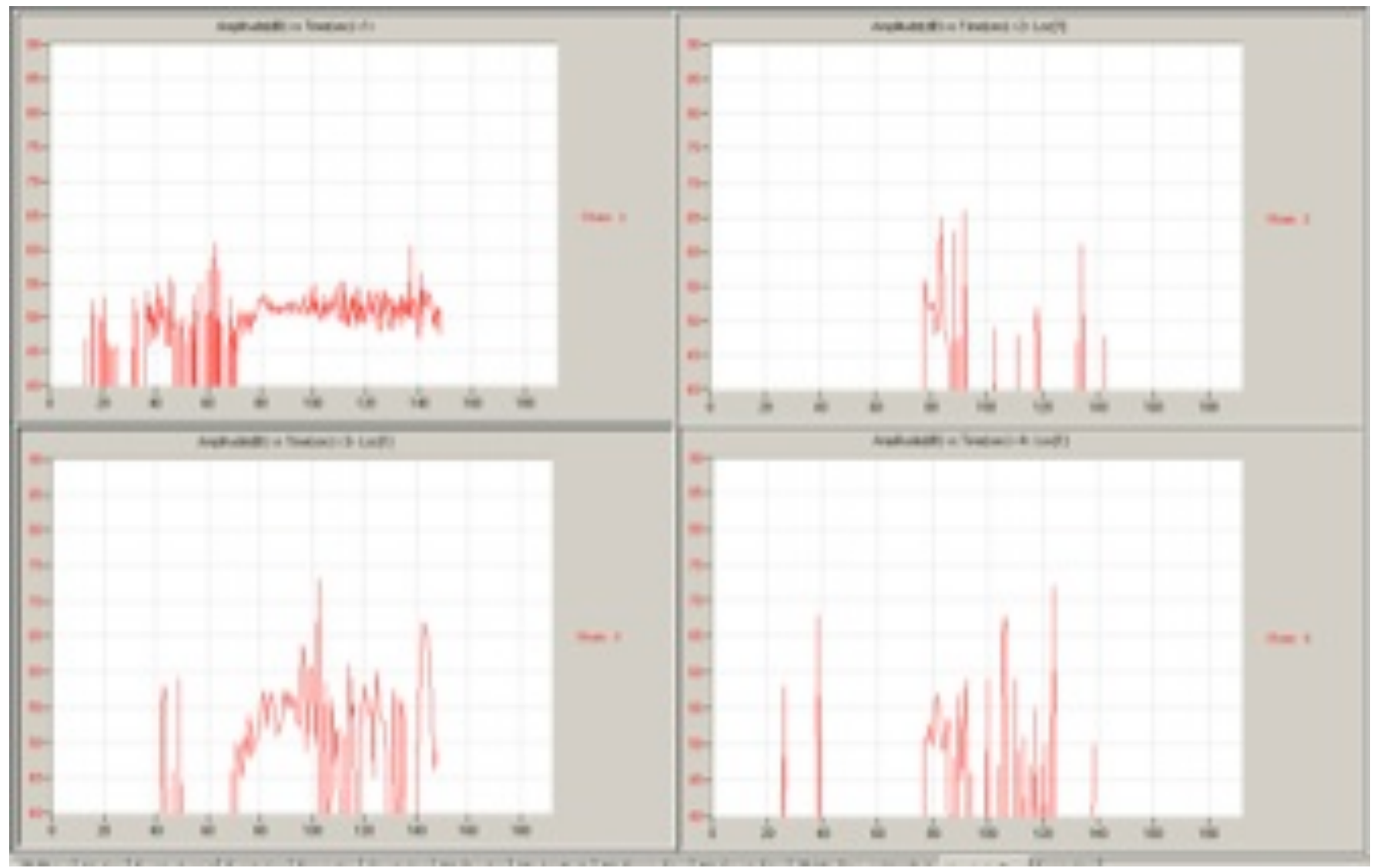

Fig. (3). Amplitude-time curve.

amplitude, but the variation can be large; channel 3 has maximum value $73 \mathrm{~dB}$ which occurs in about $103 \mathrm{~s}$; channel 4 has the maximum value about $72 \mathrm{db}$ occurring in about $124 \mathrm{~s}$. It can be seen from the 4 curves that they do not have obvious common peak moment and the signal is disordered. However, detailed conclusion still requires further analysis.

\subsubsection{Ringing-Time Curve}

Fig. (4) presents the time-varying curve of AE ringing count. This curve can roughly reflect the extent of injury to stratum and injury status in different times. It can be seen from the curve that there are several significant peaks, the first occurring in $81 \mathrm{~s}$ with a value of about 13400 with subsequent fluctuation being small; the second peak value is about 9200 , occurring in $130 \mathrm{~s}$; the maximum peak value of 15600 occurs at about $137 \mathrm{~s}$. The production mechanism of AE signal however still requires further analysis.

\subsubsection{Energy-Time (Sum, All) Curve}

Fig. (5) shows the time-varying curve of AE energy. This curve can correctly reflect the energy released from the injury of stratum in different times. It can be seen from Fig.

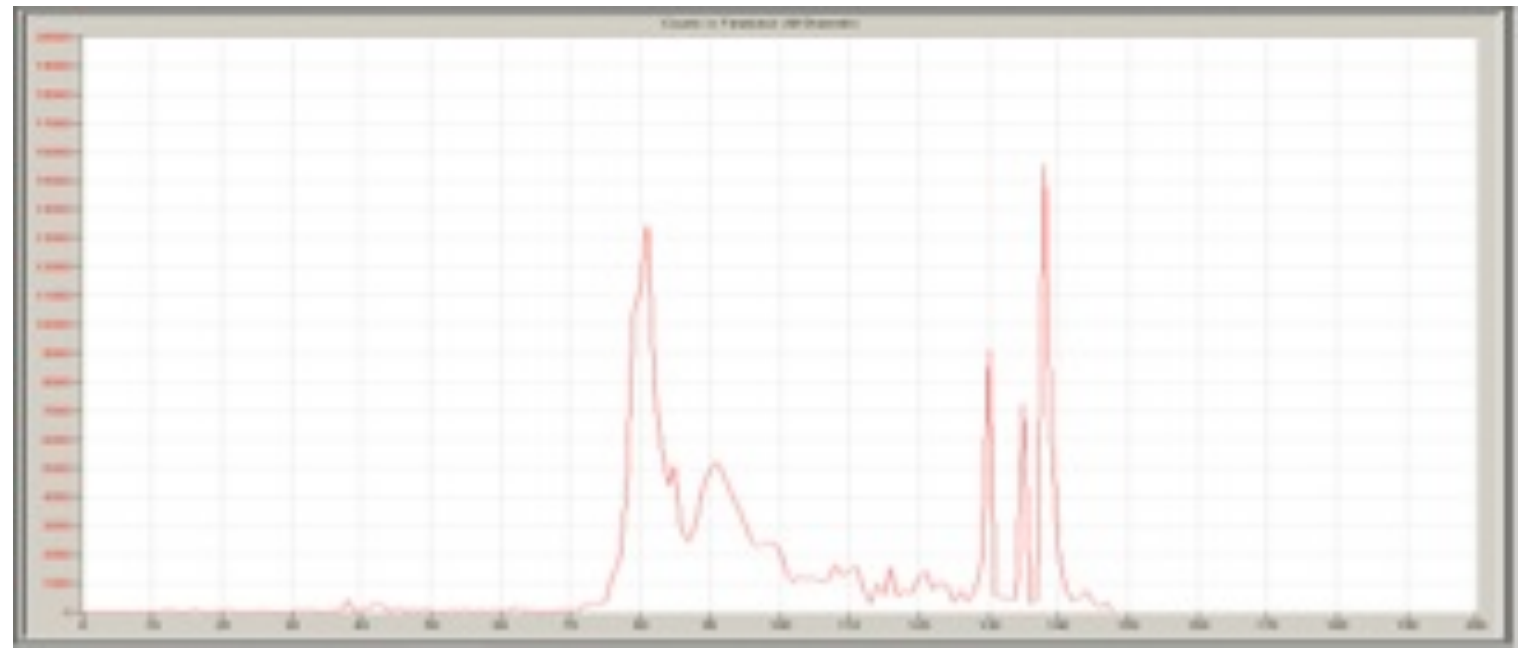

Fig. (4). Counts-time curve. 


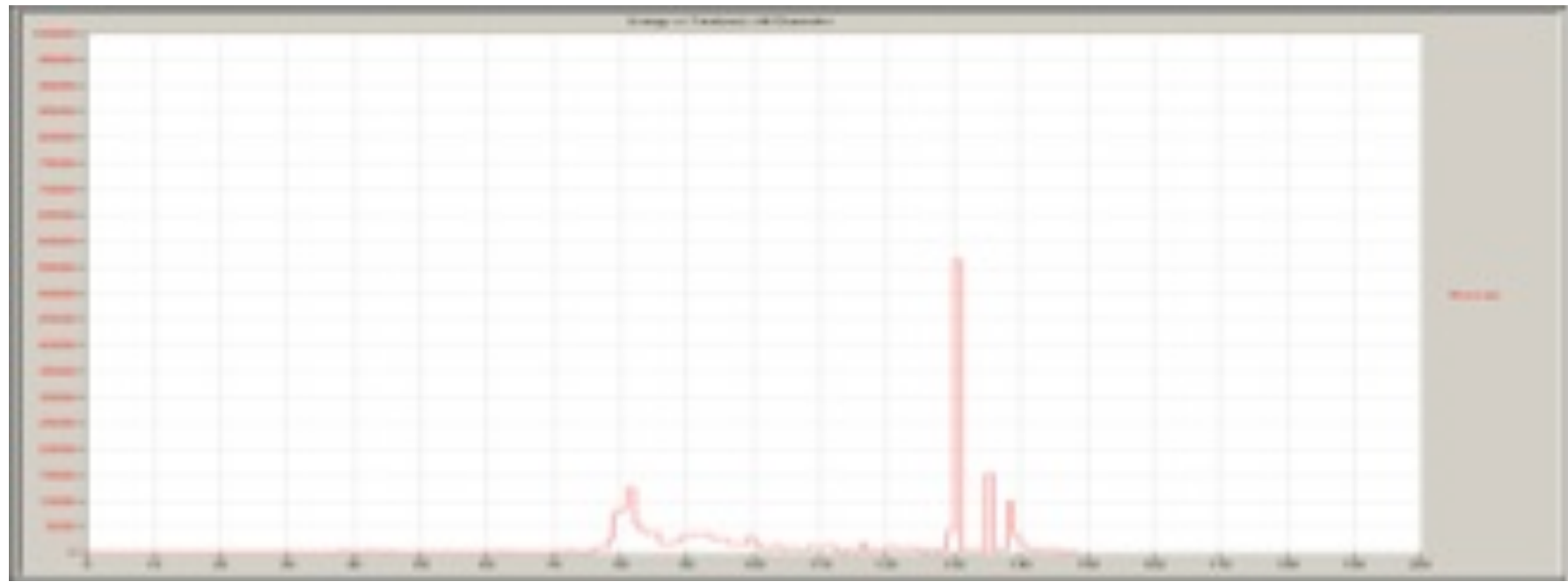

Fig. (5). Energy-time (sum, all) curve.

(5) that the occurring time of several peak values is basically consistent with Counts-Time (Sum, All) curve. What is different is that the peak value of energy curve occurs in about 130 s and is obviously greater than other peak values. Thus, it can be judged that given there is an AE information source of serious injury (there is great crack or fracture surface on simulated stratum), which must occur in 130 s. The subsequent peak values of different sizes should be the extension of stratum injury at 130 s. Concrete injury extent requires further analysis.

\subsubsection{Energy-Time (Average, Channel and All) Curve}

Fig. (6) depicts the time-varying curve of energy in each channel and average value of total energy. By these curves, one can more precisely determine the occurrence time and strength of information source of AE signal collected by each channel. It can be seen from the trend of curve in Fig. (6) that the time-varying curve of energy from channel 1, 2 and 3 always fluctuates in extremely low level and the maximum value is only $18 \mathrm{~dB}$. Differently, channel 4 exhibits

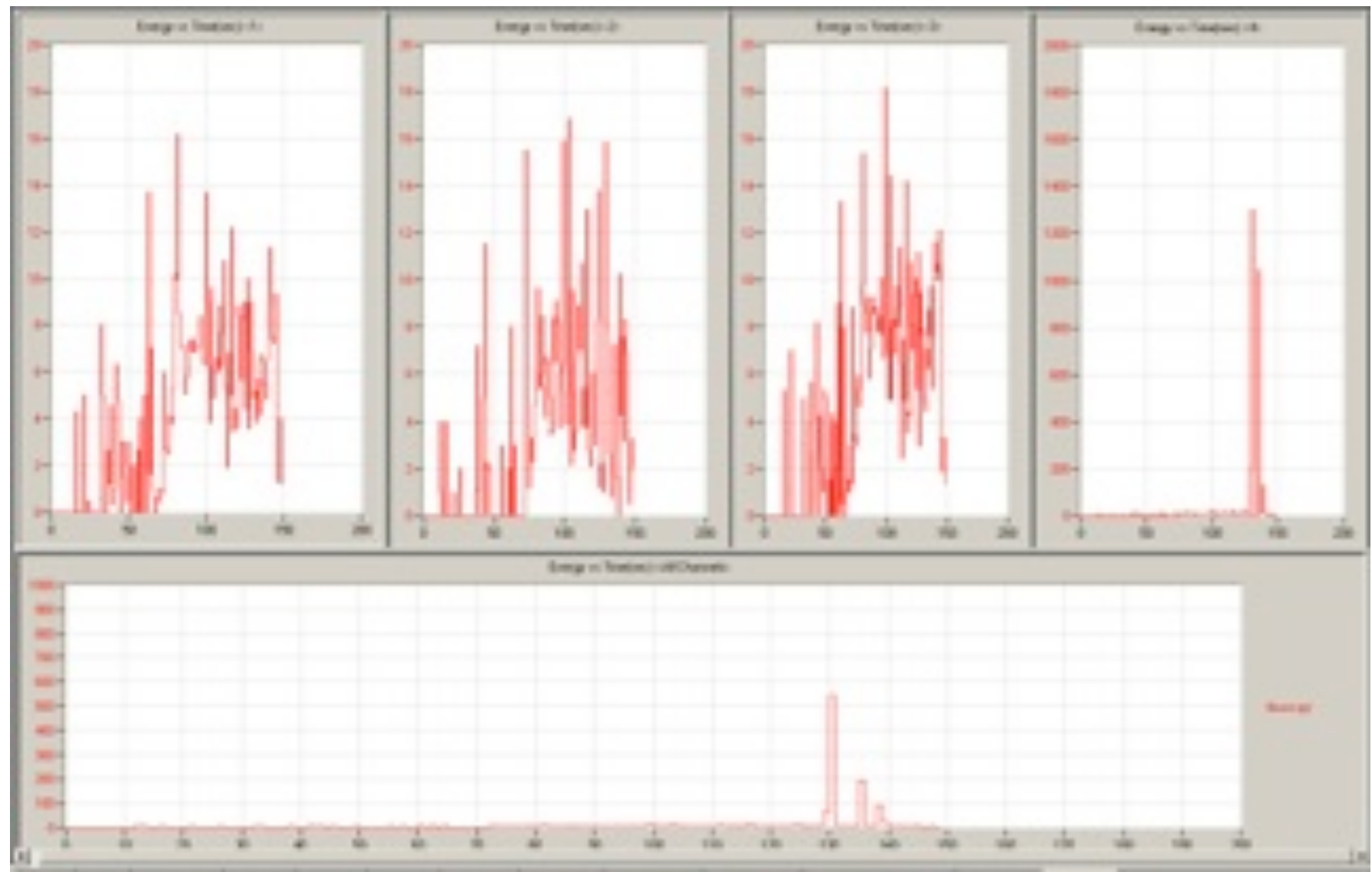

Fig. (6). Energy-time curve group. 


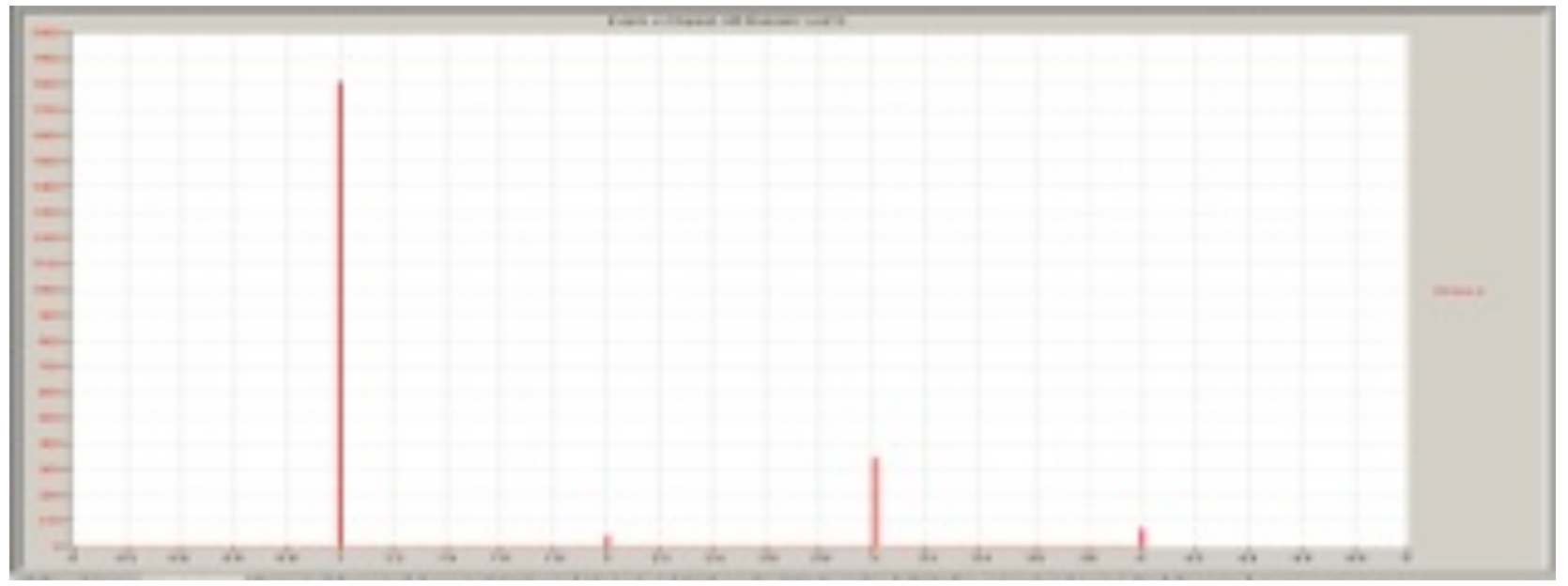

Fig. (7). Events-Channel histogram.

extremely large energy, approximately $1300 \mathrm{~dB}$ at $130 \mathrm{~s}$. The occurrence of this information source is either extremely close to sensor 4, or the result of uncertainties from nonstratum injury; from Energy-Time (Average, All) curve, in addition to the extreme peak energy at 130 s, other times show no peak value. Concrete conclusion requires further analysis.

\subsubsection{Event-Channel Histogram}

Fig. (7) is the total number of AE events collected from each channel. This image reflects the activity of each channel during the test and reflects to some extent the injury of stratum near sensors of each channel. It can be seen from histogram that the number of AE events in channel 1 and 3 is obviously greater than channel 2 and 4, which indicates that the seriously injured stratum lies in the side of sensors close to channel 1 and 3 while the number of $\mathrm{AE}$ in channel 4 is not great. This verifies the burstiness of energy peak at $130 \mathrm{~s}$.

\subsubsection{Event-Time (Sum, All) Curve}

Fig. (8) displays the AE event-time curve which reflects to some extent the damage and injury of stratum during test. It can be seen from Fig. (8) that different from the above time-varying curves of energy and ringing, the main peak value of this curve occurs in about $80 \mathrm{~s}$ and subsequent fluctuation gradually becomes small. This explains the occurrence of outburst AE source from one side. Fracture surface is probably the brittle rupture injury formed in $80 \mathrm{~s}$. Further analysis is still required.

\subsubsection{Hits, Ringing-Time Synchronous Curve}

Fig. (9) shows the time-varying curve of wave hits and ringing count. The two curves visually enable the comparison of wave hits and ringing count, and further infer the strength of AE wave hits. It can be seen from Fig. (9) that the maximum of ringing count occurs in 80 s and wave hits in about $138 \mathrm{~s}$, which is different from the peak value position (130s) of wave hits and ringing count, ringing-time curve and energy-time curve. Ringing count curve also exhibits peak value at 130 s and the ratio of ringing count and wave hit number is significantly greater than other times. On the above basis, it can be inferred that the AE wave hits at $130 \mathrm{~s}$ and $138 \mathrm{~s}$ are much stronger than 80 s and the energy is much greater. Sound emission signal at 130 s reaches to the peak while at $138 \mathrm{~s}$.

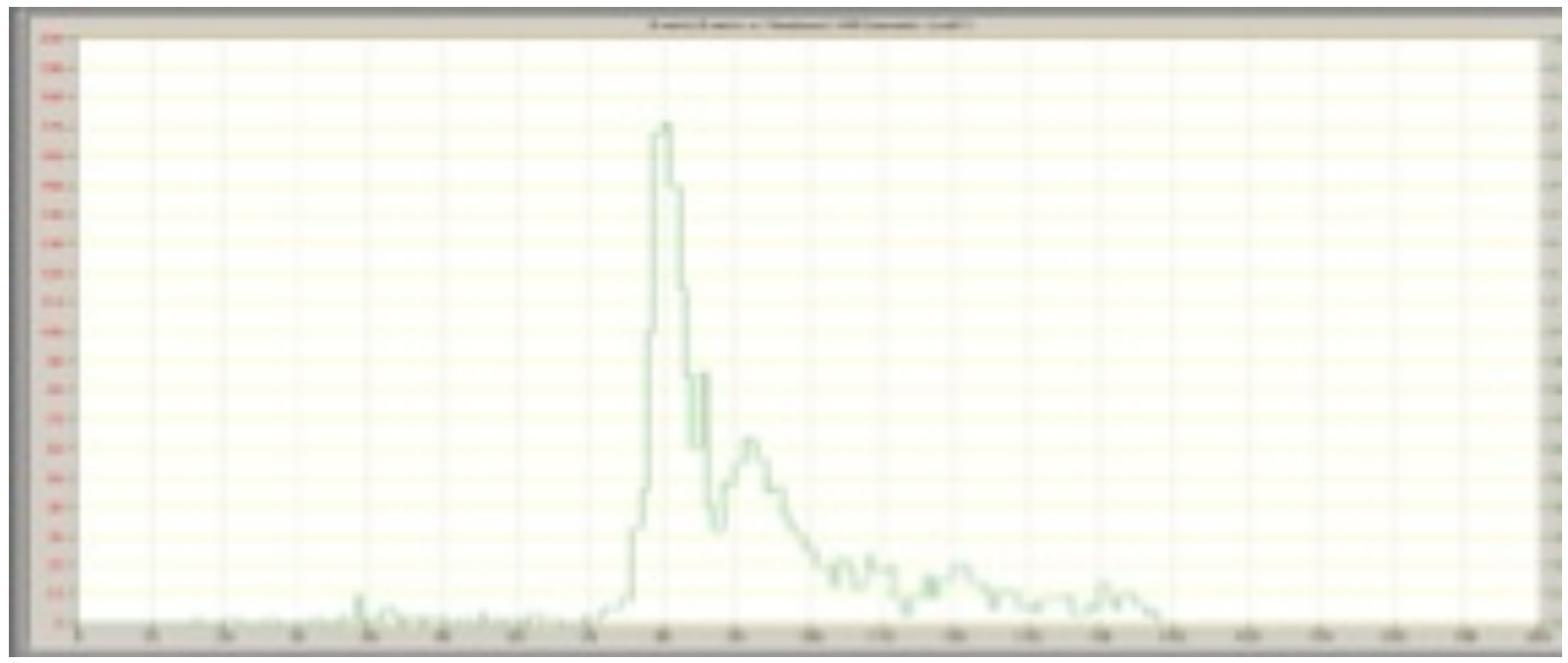

Fig. (8). Events-time curve. 


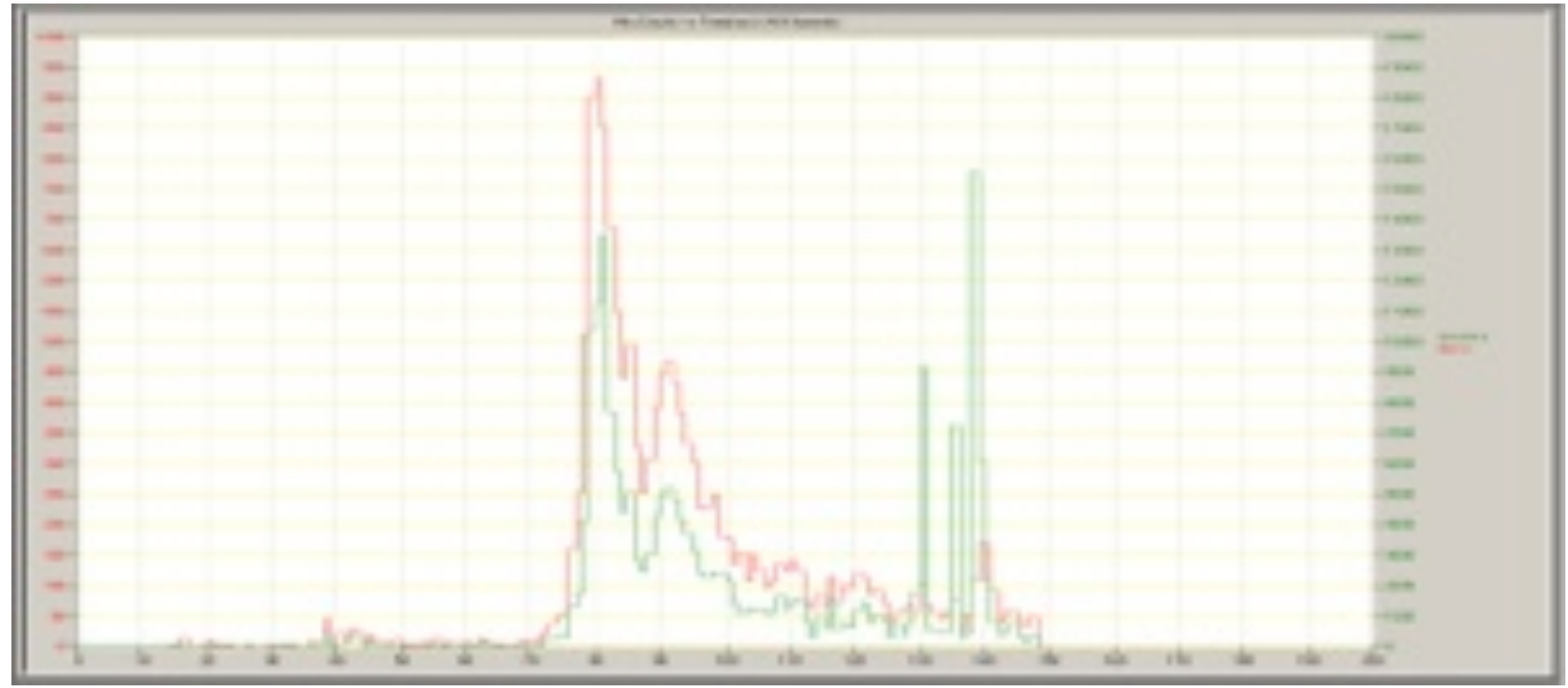

Fig. (9). Hits, counts-time synchronous curve.

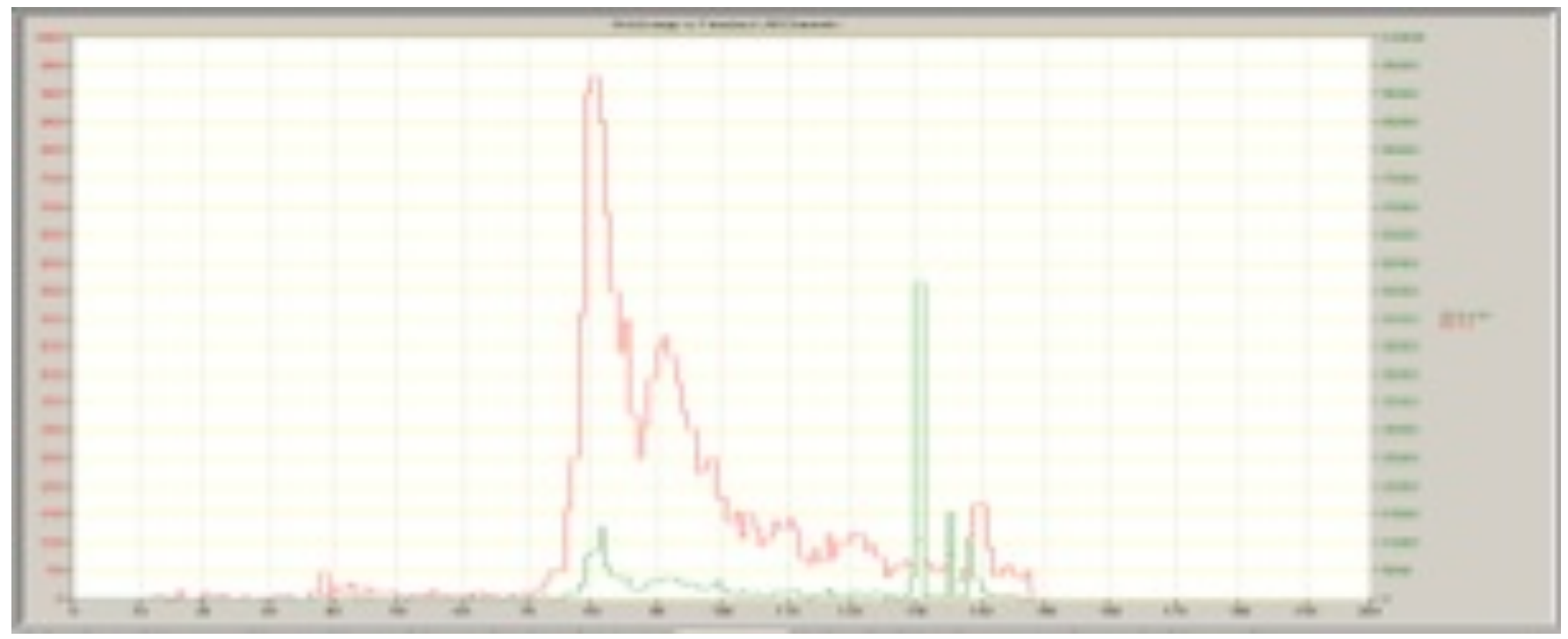

Fig. (10). Hits, energy-time synchronous curve.

\subsubsection{Wave Hits, Energy-Time Synchronous Curve}

Fig. (10) displays the time-varying curve of wave hits and energy. Fig. (10) enables visual comparison of wave hit and energy trend. According to the curve of hit number and energy, the strength of AE wave hits can be inferred. The characteristic of this curve is similar to Fig. (9). A common conclusion may be obtained.

\subsubsection{Hit-Time (Average, Channel and All) Curve Diagram}

Hits-Time (Average, Channel and All) curve Fig. (11) shows the time-varying curve of average hits and total hit average from each channel. This group of curves can visually identify the difference and association between curves by comparison and facilitate further analysis. It can be seen from grouped Fig. (11) that this group of curves has similar trend and peak value characteristics; the maximum occurs at about $80 \mathrm{~s}$. Different from other 3 channels, the channel 4 has obvious second peak at about 130s, which has been proven in the previous conclusion.

\subsubsection{Distribution Point Diagram of Y Position, Events-X Position}

Y Position and Events-X position distribution point Fig. (12) presents the distribution characteristics of AE events in test plane domain. The distribution situations of $\mathrm{AE}$ information source can be visually seen from Fig. (12). It can be seen from point diagram that sound emission events are distributed centrally, mainly in $0.4 \mathrm{~m} \sim 0.5 \mathrm{~m}$ and $0.54 \mathrm{~m} \sim 0.62$ $\mathrm{m}$ (left bottom of marble slab as origin of coordinate and panel length direction as $\mathrm{x}$ axis) two band-shaped sections. It can be judged that the injury in this section connects each other.

Based on the above analysis, the following conclusions can be obtained: At about 80s, the two band-typed sections, 


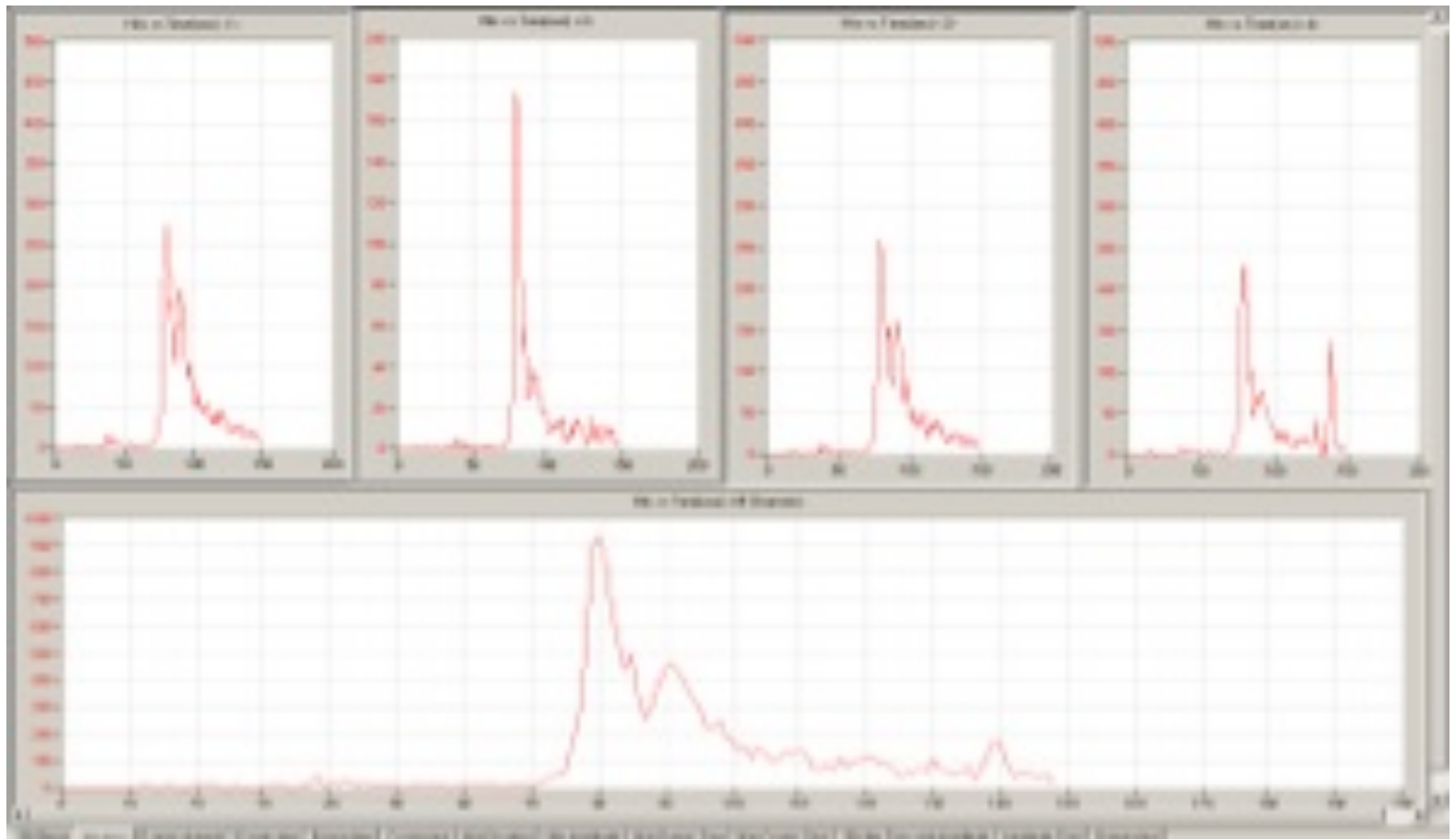

Fig. (11). Hits-time curve group.

$0.4 \sim 0.5$ and $0.54 \sim 0$. 62, suffer from penetrating injury. At the early stage of injury, AE signal has lower energy, but there are various sound emission events; in about 130s and $138 \mathrm{~s}$, the injuries in these two band-typed sections are suddenly connected forming a fracture surface.

\section{ANALYSIS ON THE RESULT OF IMAGE DIAGNOSIS ORIENTATION}

The above analysis only provides the analytical and processing process of a test document. This method is utilized to analyze the whole fracture process of a timevarying boundary roof. The result of orientation is shown in Table 2 and Fig. (13).

The orientation test of whole stratum can be classified into 7 test stages. From the orientation result (Table 2 and Fig. 13) of the whole stratum, it is observed that there are 5 fracture surfaces on the whole stratum named as No.1, 2, 3, 4 and 5 from left to right. The No.2 and 3 fracture surface occurs in the first test stage. From Table 2, test stage 6 also sets place for No.2 fracture surface. There are two reasons

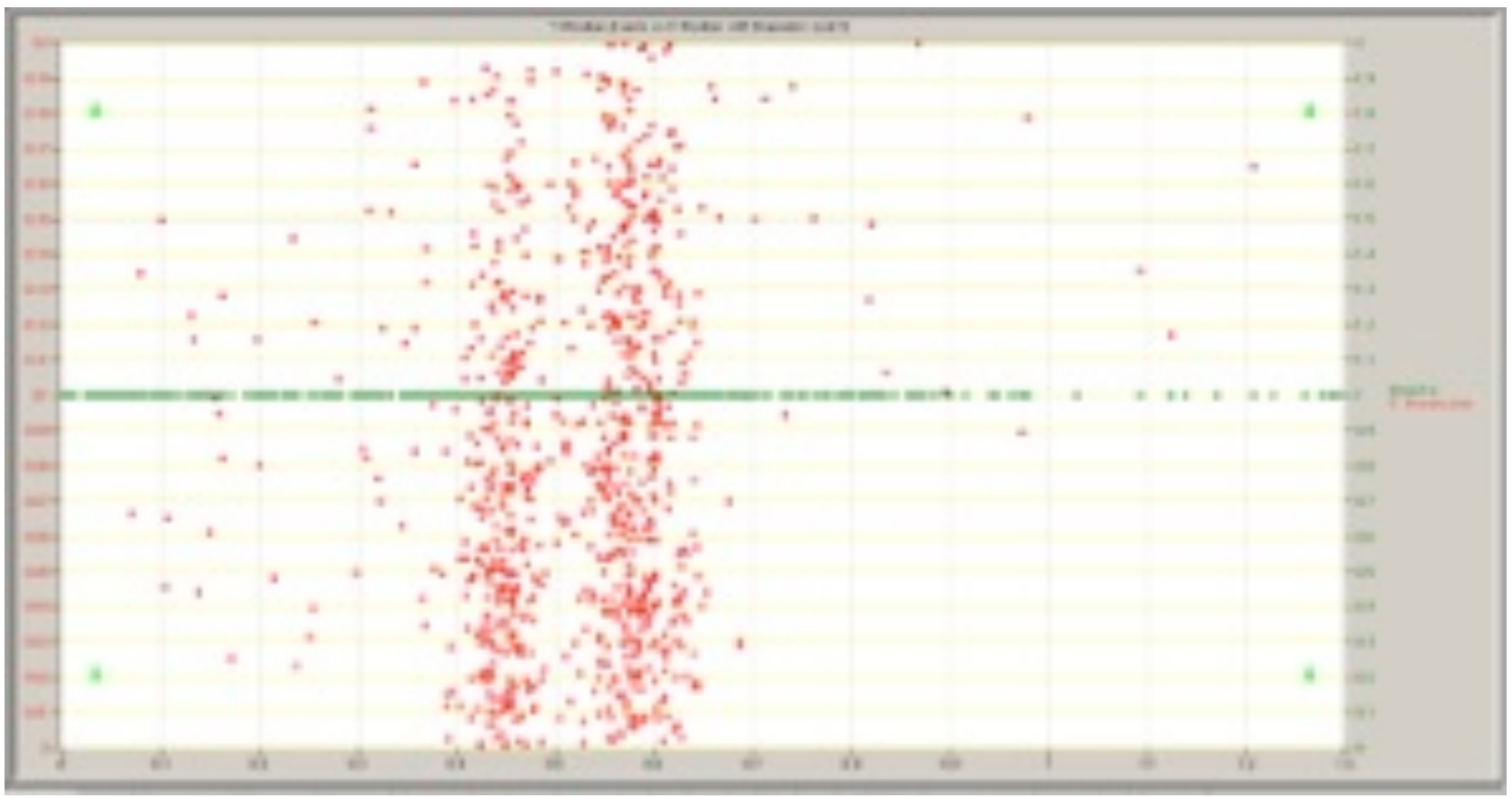

Fig. (12). Y Position, Events-X position scatter figure. 
Table 2. Test the cross section and the actual cross-section position contrast table.

\begin{tabular}{|c|c|c|c|c|}
\hline $\begin{array}{c}\text { Oriented fracture } \\
\text { surface area }\end{array}$ & $0.2 \sim 0.35$ & $0.4 \sim 0.5$ & $0.54 \sim 0.62$ & $0.7 \sim 0.8$ \\
\hline $\begin{array}{c}\text { Center line of } \\
\text { orientation area }\end{array}$ & 0.28 & 0.45 & 0.58 & 0.75 \\
\hline $\begin{array}{c}\text { Orientation } \\
\text { testing stage }\end{array}$ & 7 & 1,6 & 1 & 3 \\
\hline $\begin{array}{c}\text { Actual fracture } \\
\text { surface position }(\mathrm{m})\end{array}$ & 0.31 & 0.48 & 0.59 & $0.66 \sim 0.76$ \\
\hline
\end{tabular}

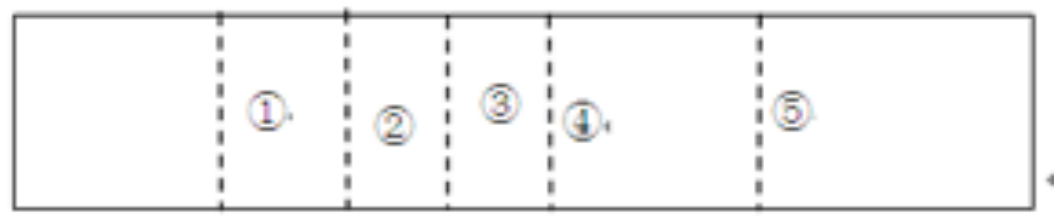

\section{a) $\mathrm{AE}$ orientation test result}

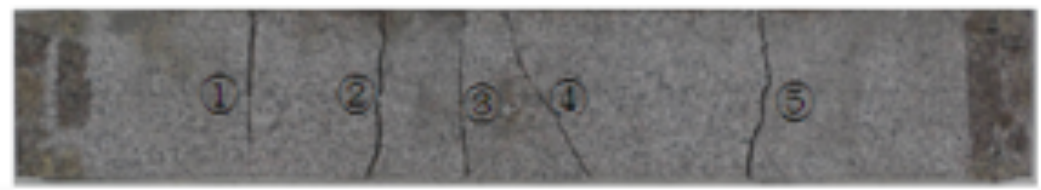

b) Actual fracture surface

Fig. (13). The experimental results show figure.

for that: 1) No.2 fracture surface is not fully formed in the first stage and finally runs through in stage 6. 2) Fracture surface has been fully penetrated in the first stage and orientation result occurs in stage 6 , which is caused by the mutual friction between sections. No.5 fracture surface also possesses similar situations. It can be seen from Fig. (13) that there are also 5 actual fracture surfaces, which conforms with the number of fracture surfaces for orientation test.

\begin{tabular}{|l|l|l|l|l|}
\hline & & & & \\
\hline & & & & \\
\hline & & & & \\
\hline & & & & \\
\hline
\end{tabular}

\section{CONCLUSION}

This paper utilized self-designed time-varying boundary sound emission test system for rock stratum fracture to complete the sound emission orientation test upon the fracture of marble slab. A new method-image diagnosis and orientation method is proposed to locate fracture section. Sound emission signal Y Position and Events-X position distribution point diagram is utilized to determine the position of fracture surface. By analyzing the time-varying curve of Counts, Energy and Hits of acoustic emission signal in the same stage, the occurrence moment of rock stratum fracture section was confirmed and the development mechanism of fracture section was analyzed. Through study, the following conclusion is obtained:

(1) Actual fracture surface is $100 \%$ inclusive in the orientation area.

(2) Oriented fracture surface position is close to actual fracture surface. Sound emission image diagnosis technique is featured by high orientation precision, small error, and excellent orientation effect of sound emission.

(3) During sound emission test, existing friction between fracture surfaces may produce a great many strong sound emission signals resulting in multiple orientations of fracture surfaces and misleading analysis.

(4) One test stage may have multiple fracture surfaces which can be precisely oriented by image diagnosis orientation method.

(5) During orientation test, it isa must to make a comprehensive orientation analysis on test objects and master the whole process of orientation test to give more precise orientation judgment and provide reliable assistance and technical supports for actual decision-making and prevention of mining engineering disaster. 
(6) Orientation result indicates that sound emission image diagnosis orientation method has excellent maneuverability and practicability.

\section{CONFLICT OF INTEREST}

The authors confirm that this article content has no conflict of interest.

\section{ACKNOWLEDGEMENTS}

This work has been supported by the National Program on Key Basic Research Project (973 Program) (NO. 2013CB227900); the state "1025" science and technology support projects (NO. 2012BAB13B00).

\section{REFERENCES}

[1] H. Man-chao, X. He-ping, P. Su-ping, and J. Yao-dong, "Study on rock mechanics in deep mining engineering", Chinese Journal of Rock Mechanics and Engineering, vol. 16, pp. 2803-2813, 2005.
[2] H. Man-chao, X. He-ping, P. Su-ping, and J. Yao-dong, "Study on crack dynamic propagation process of rock samples based on aconstic emission location,"Chinese Journal of Rock Mechanics and Engineering, vol. 5, pp. 5944-950, 2007.

[3] Z. Ya-bin, Z. Yu-cheng, and W. Zi-bo, S "Study on broken law of roof above coal mining face under influences of fault," Coal Engineering, vol. 9, pp. 71-74, 2013.

[4] Y. Guochao, D. Chunsheng, and M.A. Zhonghu, "Application of acoustic emission technology in mining stope key stratum in overhead mining," Journal of Liaoning Technical University, vol.1, pp. 9-12, 2010.

[5] L. Zhong-yu, G. Feng, Z. Wei-ping, and X.Xiao-li, "Experimental analysis on acoustic emission of fracture rock,"Mining Engineering, vol. 5, no. 2, pp. 16-17, 2007.

[6] H. Bing-xiang, L. Chang-you, and X. Jia-lin, "Research on through degree overlying strata fracture fissure induced by mining,"Journal of China University of Mining \& Technology, vol. 1, pp.45-49, 2010.

[7] Z. Jian-ping, P. Jian-liang, and L. Jian-feng, "Inverstigation on acoustic emission behaviour its time-space evolution mechanism in failure process of coal-rock combined body," Chinese Journal of Rock Mechanics and Engineering, vol. 8, pp. 1564-1570, 2011.

[8] L.Xiao-yin, G. Feng, and Wei-ping, "Analysis of fracturing mechanism of stope roof based on plate model," Journal of Mining \& Safety Engineering, vol. 2, pp. 180-183,2008. 